\title{
Erratum to: Textbook of Ocular Trauma
}

Stephen C. Kaufman and Douglas R. Lazzaro

Erratum to: S.C. Kaufman and

D.R. Lazzaro (eds.), Textbook of Ocular Trauma, DOI 10.1007/978-3-319-47633-9

The original version of the book was inadvertently published without volume editor name "Stephen C. Kaufman" which has to be included in the "Contributors" list of FM. The erratum book has been updated with the change.

The updated online version of the book can be found at http://dx.doi.org/10.1007/978-3-319-47633-9 